\title{
Vinylogous hydrazone activation in stereoselective synthesis of 2,3-dihydro-1 H-pyrrolizines - an organocatalytic, metal-free route to ketorolac
}

\author{
Justyna Kowalska, ${ }^{[a]}$ Beata Łukasik, ${ }^{[a]}$ Sebastian Frankowski, ${ }^{[a]}$ Lesław Sieroń, ${ }^{[b]}$ and Łukasz Albrecht $^{*[a]}$
}

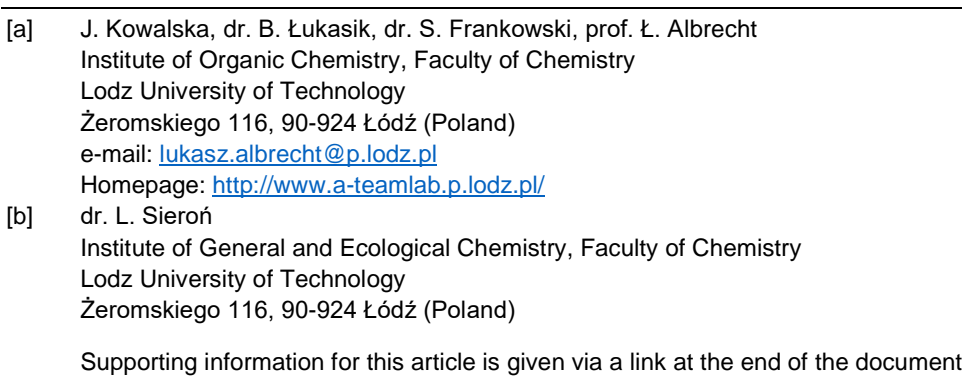

\begin{abstract}
In this paper, the application of organocatalysis in the synthesis of 2,3-dihydro-1H-pyrrolizines is demonstrated. $\alpha, \beta$ Unsaturated aldehydes and pyrrole-based hydrazone readily participated in the formal $(3+2)$-cycloaddition that was realized according to aminocatalytic iminium ion activation. Products were obtained in high chemical yields, with excellent stereocontrol and selected additional transformations were also presented. The developed method was utilized in the first organocatalytic synthesis of ketorolac, a non-steroidal anti-inflammatory drug.
\end{abstract}

\section{Introduction}

Contemporary asymmetric synthesis has become one of the most exploited branches of organic synthesis. ${ }^{[1-4]}$ Within this field, organocatalysis ${ }^{[2]}$ has evolved into an important method being complementary to chiral metal complexes ${ }^{[3]}$ and biocatalysis. ${ }^{[4]}$ It provides access to numerous chiral building blocks using optically pure organic compounds as catalysts. Aminocatalysis employs secondary or primary amines for the stereoselective functionalization of carbonyl compounds with the enamine and iminium-ion activations being the most fundamental modus operandi (Figure 1, top). ${ }^{[5]}$ These methodologies already proved their usefulness in the synthesis of natural products and pharmaceuticals e.g. oseltamivir, telcagepant, paroxetine, tolterodine or cispentacin (Figure 1, bottom) with aminocatalytic step being responsible for the stereoselectivity of a given approach. ${ }^{[6]}$

Ketorolac is a non-steroidal anti-inflammatory drug, which is sold under the brand name Toradol. In 2018 it was the $194^{\text {th }}$ most commonly prescribed pharmaceutical in the United States. ${ }^{[7]}$. Although ketorolac is marketed as a racemic mixture, the $(S)$ enantiomer is much time more potent than its $(R)$-enantiomer, therefore the enantioselective synthesis of this molecule is of importance. ${ }^{[8]}$

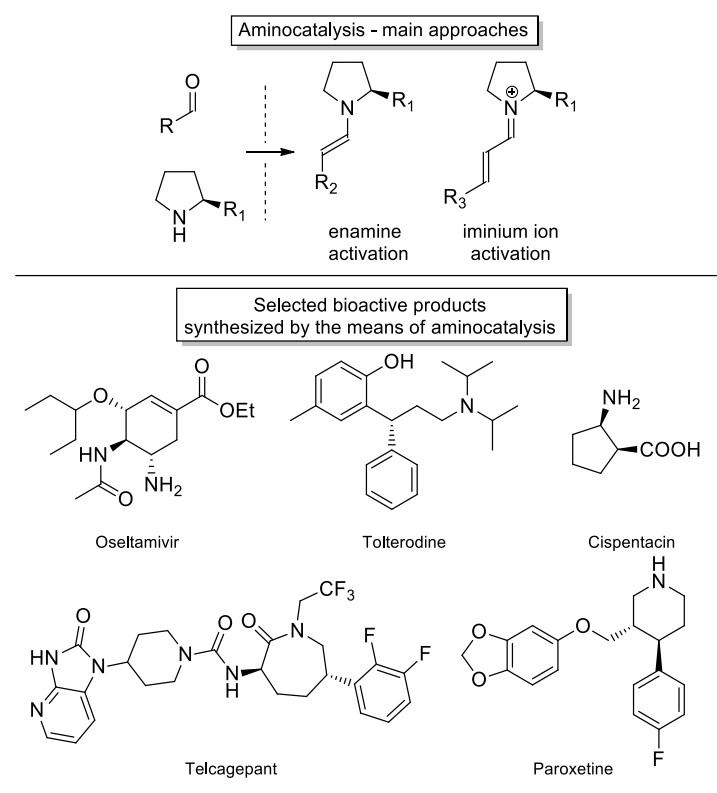

Figure 1. Asymmetric organocatalysis - fundamental activation strategies and their applications in the synthesis of bioactive products.

Although there are numerous methods of the synthesis of racemic ketorolac relying mainly on the cyclization reactions, ${ }^{[9 a-c]}$ the protocols for its preparation in enantiomerically enriched form are limited and involve: 1) separation of enantiomers by the derivatization combined with chromatography methods; ${ }^{[9 d]}$ 2) enzymatic kinetic resolution of racemate $;{ }^{[9 e, f]} 3$ ) utilization of the chiral auxiliary approach; ${ }^{[9 \mathrm{~g}, \mathrm{~h}]}$ and 4 ) enantioselective cyclization catalyzed by chiral gold complex ${ }^{[i]}$ (Scheme 1). 


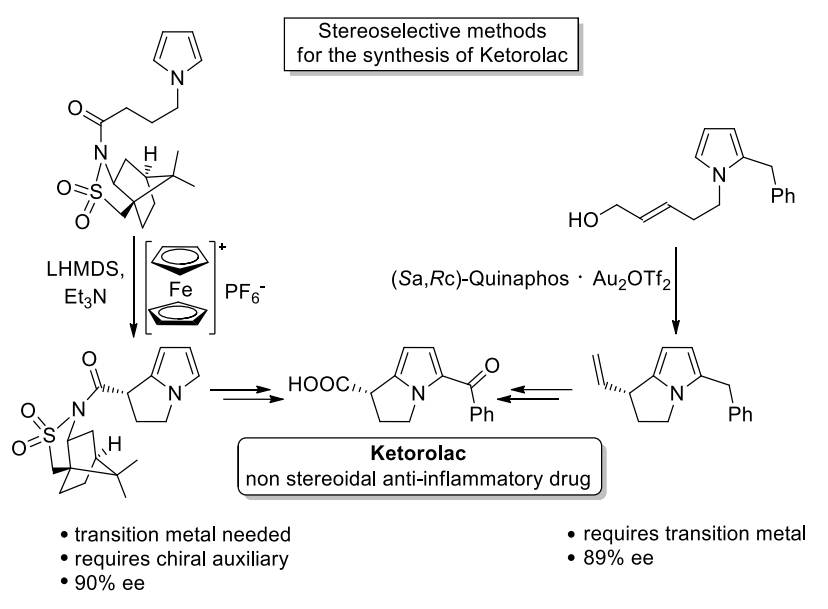

Scheme 1. Stereoselective syntheses of ketorolac.

Umpolung can be defined as the reversal of the reactivity of a given functional group by appropriate chemical modification of its structure. The development of such strategic methodologies receives considerable interest from the community as it constitutes a unique tools for the identification of non-classical synthetic pathways. Most explored approaches allowing for the umpolung of the carbonyl moiety include $\mathrm{NHC}$ catalysis ${ }^{[10]}$ and hydrazone umpolung (Scheme 2, top). ${ }^{[11]}$ In the latter, the appropriate carbonyl compound is reacted with hydrazine derivative providing hydrazone which possesses reversed reactivity, changing the character of the carbonyl group to nucleophilic. Recently, we have demonstrated that hydrazones derived from heteroaromatic aldehydes readily participate in the asymmetric Friedel-Crafts reaction with $\alpha, \beta$-unsaturated aldehydes performed under iminium ion activation (Scheme 2, middle). Notably, in this transformation the hydrazone umpolung effect was efficiently transmitted over the heteroaromatic system providing a unique opportunity for the functionalization of heteroaromatic frameworks. ${ }^{[11 f]}$

Given the importance of heteroaromatic compounds in the contemporary organic and medicinal chemistry, studies on the application of vinylogous hydrazone umpolung activation for the synthesis of 2,3-dihydro-1H-pyrrolizine core were undertaken. Notably, such structural motif is present in various biologically active molecules such as ketorolac, ${ }^{[12 a]}$ dehydroretronecine, ${ }^{[12 b]}$ or licofelone. ${ }^{[12 c]}$ It was anticipated that hydrazone $\mathbf{1}$ derived from $1 \mathrm{H}$-pyrrole-2-carbaldehyde should act as 1,2-dinucleophilic component capable of participating in annulative processes with appropriately activated 1,3-dielectrophilic reagents, thus providing access 2,3-dihydro- $1 \mathrm{H}$-pyrrolizine via (3+2)cycloaddition. $\alpha, \beta-$ Unsaturated aldehydes 2 were selected as reaction partners as their iminium-ion activation constitutes a reliable method in asymmetric catalysis. Furthermore, functional groups introduced following such a strategy possess strong synthetic potential as confirmed in the first organocatalytic synthesis of ketorolac.
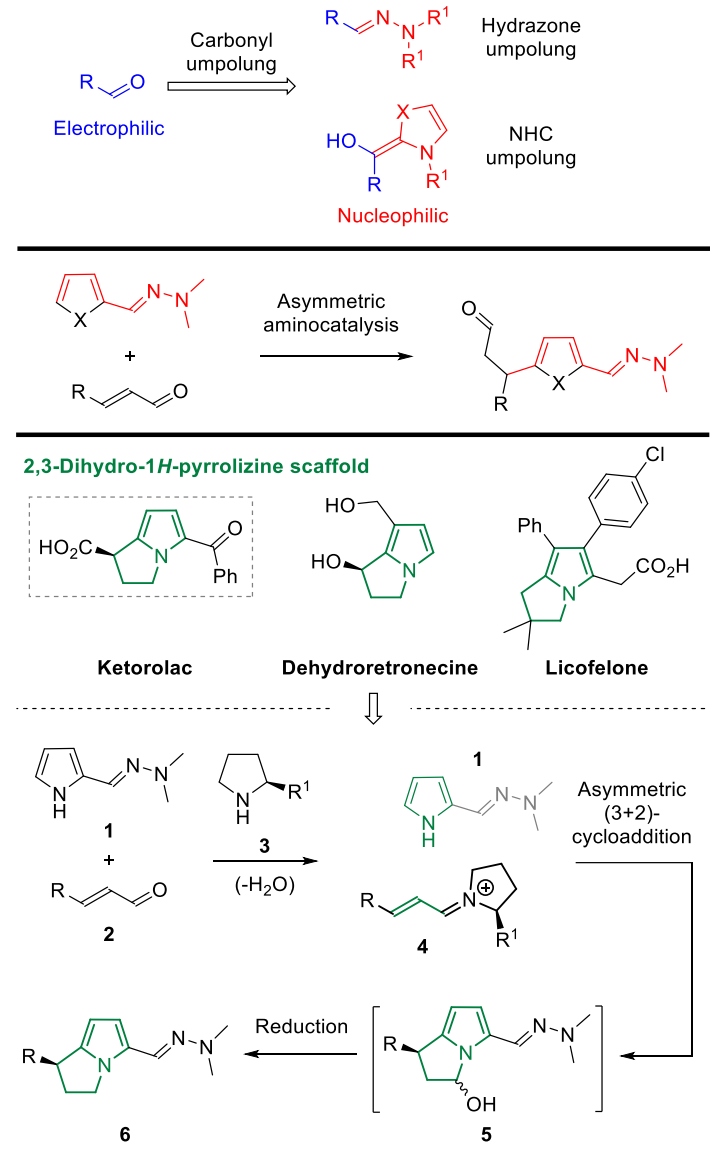

Scheme 2. Application of vinylogous hydrazones in the synthesis of 2,3dihydro- $1 \mathrm{H}$-pyrrolizines.

Herein we present our studies on highly stereoselective aminocatalytic cascade reaction between $\alpha, \beta$-unsaturated aldehydes 2 and hydrazone 1 derived from $1 \mathrm{H}$-pyrrole-2carbaldehyde leading to 2,3-dihydro- $1 \mathrm{H}$-pyrrolizine derivatives 6 with excellent results (Scheme 2, bottom). Moreover, in the manuscript the application of the elaborated methodology in the enantioselective synthesis of ketorolac has been developed, thus establishing the first organocatalytic approach for the synthesis of this biologically active molecule.

\section{Results and Discussion}

Optimization studies were performed using (E)-2-((2,2dimethylhydrazono)methyl)- $1 H$-pyrrole $\mathbf{1}$ and transcinnamaldehyde $\mathbf{2 a}$. Initial experiments were carried out in dichloromethane, in the presence of Jørgensen's catalyst 3a and trifluoroacetic acid as co-catalyst at $-20{ }^{\circ} \mathrm{C}$. To our delight, the devised cycloaddition proceeded smoothly providing product $\mathbf{5 a}$ as a diastereomeric mixture. In order to determine the enantioselectivity of the process the obtained hemiaminal $\mathbf{5 a}$ was directly reduced to $6 a$ via one-pot procedure using triethylsilane and boron trifluoride diethyl etherate. Amine $6 \mathbf{a}$ was isolated in good yield, however, the stereoselectivity was unsatisfactory (Table 1, entry 1). Nonetheless, further catalyst screening indicated catalyst $\mathbf{3} \mathbf{a}$ as the most optimal in terms of enantioselectivity of the reaction (Table 1, compare entries 1-5). 
Subsequently, various solvents were tested (Table 1, entries 1, 6-9) revealing toluene as the most suited medium for this transformation (Table 1, entry 9). The influence of acid additive was also investigated (Table 1, entry 9-12). Among examined acids, monochloroacetic acid (MCA) turned out to be the most beneficial (Table 1, entry 12). Subsequently, the influence of catalyst loading (Table 1, entry 13) and concentration (Table 1, entry 14-15) on the cascade were tested, but no improvement was observed. Finally, it was found that lowering the temperature to $-40{ }^{\circ} \mathrm{C}$ had crucial impact on the stereochemical outcome of the reaction (Table 1, entry 16 ) providing product $6 \mathbf{a}$ with high enantioselectivity and satisfactory yield, thus establishing final reaction conditions.

Table 1. Asymmetric synthesis of 2,3-dihydro- $1 \mathrm{H}$-pyrrolizine $\mathbf{6 a}$ - optimization studies. ${ }^{[a]}$

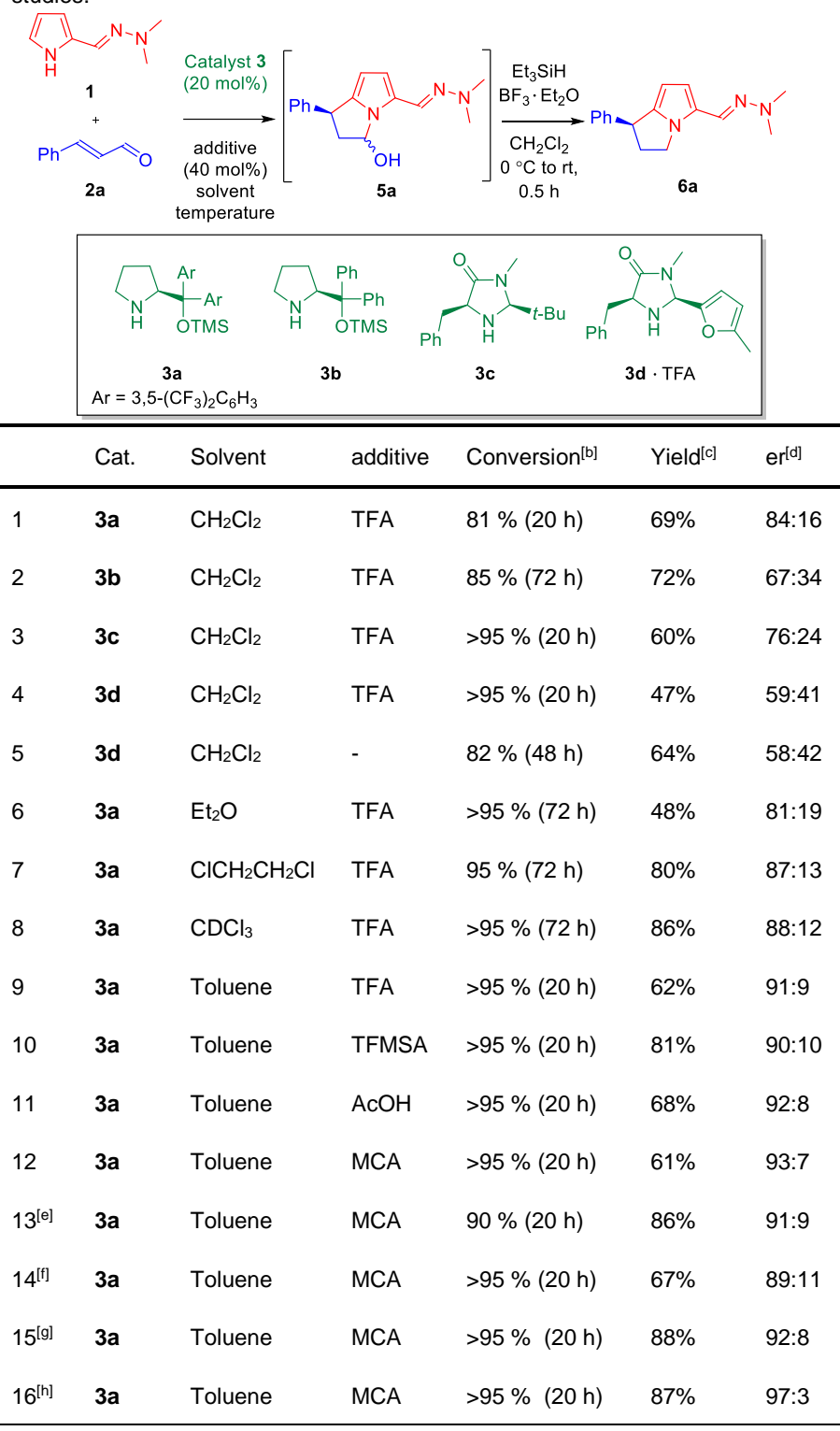

[a] Reactions performed on a $0.05 \mathrm{mmol}$ scale using 1 (1.2 equiv) and 2a (1.0 equiv.) in $0.2 \mathrm{~mL}$ of the solvent for $20 \mathrm{~h}$ at $-20{ }^{\circ} \mathrm{C}$ (for full screening account, see Supporting Information). [b] Conversion as determined by ${ }^{1} \mathrm{H}$ NMR of a crude reaction mixture. Time necessary to obtain the indicated conversion is given in parentheses. [c] Isolated yield of $6 \mathbf{a}$ after the column chromatography is given. [d] Determined by a chiral stationary phase UPC ${ }^{2}$. [e] Reaction performed using $10 \mathrm{~mol} \%$ of catalyst 3a. [f] Reaction performed in $0.4 \mathrm{~mL}$ of the solvent. [g] Reaction performed in $0.1 \mathrm{~mL}$ of the solvent. [h] Reaction performed at $-40{ }^{\circ} \mathrm{C}$

With the optimal reaction conditions established (Table 1, entry $16)$, the substrate scope of the reaction was explored with various $\alpha, \beta$-unsaturated aldehydes 2 (Table 2 ). Initially, enals 2 bearing substituent at different positions of the phenyl ring and of different electronic properties were investigated (Table 2, entries 2-5). To our delight, all reactions proceeded smoothly affording target products 6 with high enantioselectivities. When para- and ortho-methoxycinnamaldehyde $\mathbf{2 b}$ and $\mathbf{2 d}$ were applied, a slightly lower enantioselectivities were observed (Table 2, entries 2,4), but the yield remained excellent. On the other hand, the use of meta-methoxycinnamaldehyde 2c resulted in a significant decrease of the yield, while maintaining its high enantiomeric excess (Table 2, entry 3 ). To our delight, enal $2 \mathrm{e}$ bearing the nitro group on the aromatic ring and 2-furylsubstituted $\alpha, \beta$-unsaturated aldehyde $2 f$ worked well under the optimal reaction conditions providing products $6 \mathbf{e}$ and $\mathbf{6 f}$ with high enantioselection, however, in the case of heteroaromatic aldehyde $2 f$ lower yield was observed (Table 2, entries 5-6). Furthermore, the possibility to introduce aliphatic substituents in the $\beta$-position of aldehydes 2 was investigated. Structurally diversified aldehydes $\mathbf{2 g}$-I were utilized in the cascade providing expected products $\mathbf{6 g}-\mathbf{i}$ in high yields and enantioselectivities (Table 2, entries 7-9). It is worth to note that enals $\mathbf{2} \mathbf{j}$ and $\mathbf{2 k}$ bearing functional groups in their side chain turned out to be excellent reactants and the desired products $\mathbf{6 j - k}$ were obtained efficiently and in a highly stereoselective manner (Table 2, entries 10-11).

Table 2. Asymmetric synthesis of 2,3-dihydro- $1 H$-pyrrolizines - $\alpha, \beta$ unsaturated aldehydes 2 scope. ${ }^{[a]}$

\begin{tabular}{|c|c|c|c|}
\hline $\begin{array}{c}1 \\
+ \\
2\end{array}$ & $\begin{array}{r}3 \mathrm{a} \\
\mathrm{ClCH}_{2} \mathrm{C} \\
\text { tolue } \\
-4 \\
2) \\
\mathrm{B} \\
\mathrm{CH}_{2} \mathrm{Cl}_{2} \text {, }\end{array}$ & $\begin{array}{l}\text { l) } \\
\% \mathrm{~mol}) \\
\mathrm{t}, 0.5 \mathrm{~h}\end{array}$ & 6 \\
\hline Entry & $\mathrm{R}$ & Yield [\%] & $\mathrm{er}^{[b]}$ \\
\hline 1 & $\mathrm{Ph}$ & 87 & $97: 3$ \\
\hline 2 & 4- $\mathrm{MeOC}_{6} \mathrm{H}_{4}$ & 78 & $92: 8$ \\
\hline 3 & 3- $\mathrm{MeOC}_{6} \mathrm{H}_{4}$ & 35 & $98: 2$ \\
\hline 4 & $2-\mathrm{MeOC}_{6} \mathrm{H}_{4}$ & 91 & $94: 6$ \\
\hline 5 & $4-\mathrm{NO}_{2} \mathrm{C}_{6} \mathrm{H}_{4}$ & 63 & $98: 2$ \\
\hline 6 & 2-Furyl & 37 & $98: 2$ \\
\hline 7 & $\mathrm{Me}$ & 59 & $97: 3$ \\
\hline 8 & $n \mathrm{Pr}$ & 89 & $99: 1$ \\
\hline 9 & Z-3-Hexenyl & 72 & $97: 3$ \\
\hline 10 & $\mathrm{BnOCH}_{2}$ & 72 & $>99$ \\
\hline 11 & $\mathrm{BzOCH}_{2}$ & 68 & $99: 1$ \\
\hline
\end{tabular}


[a] Reactions performed on a $0.1 \mathrm{mmol}$ scale using 1 (1.2 equiv.) and 2 (1.0 equiv.) in $0.4 \mathrm{~mL}$ of the solvent for $24 \mathrm{~h}$. [b] Determined by a chiral stationary phase UPC ${ }^{2}$

In the course of further studies, the possibility to transform $\mathbf{6 k}$ into the corresponding nitrile 7 was demonstrated (Scheme 3). Therefore, 6k was subjected to the reaction with magnesium bis(monoperoxyphthalate) hexahydrate. The reaction was carried out in methanol at $0{ }^{\circ} \mathrm{C}$ for 4 hours affording the corresponding nitrile 7 in $77 \%$ yield with fully maintained enantiomeric composition.

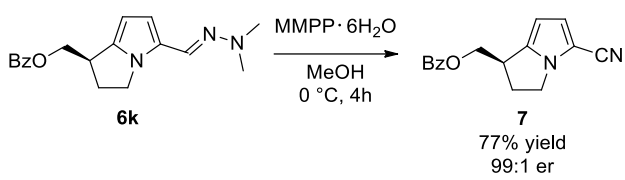

Scheme 3. Unmasking of the hydrazone moiety in $\mathbf{6 k}$.

The absolute configuration of product $\mathbf{6} \mathbf{b}$ was assigned by $\mathbf{a}$ single crystal X-ray analysis (for details, see ESI). ${ }^{[13]}$ The stereochemistry of the remaining products $6 \mathbf{a}-\mathbf{k}$ was assigned by analogy. Notably, the observed stereochemical reaction outcome is in accordance with well-established iminium ion activation of $\alpha, \beta$-unsaturated aldehydes employing diarylprolinol silyl ethers as catalysts. ${ }^{[14]}$

With the method for the enantioselective construction of 2,3dihydro- $1 \mathrm{H}$-pyrrolizine skeleton, its application for the synthesis of enantiomerically pure ketorolac was developed (Scheme 4). A one-pot reaction sequence involving aminocatalytic asymmetric (3+2)-cycloaddition between hydrazone 1 and 4benzoyloxycrotonaldehyde $\mathbf{2} \mathbf{k}$ followed by the reduction of the initially formed hemiaminal provided $\mathbf{6 k}$ with excellent enantioselectivity. It's worth to note that this step was readily scalable and realized in $2 \mathrm{mmol}$ scale (for details, see SI). Furthermore, the catalyst loading was reduced to $2 \mathrm{~mol} \%$ increasing the practicality of the approach. With the key enantioselective step accomplished, the unmasking of the hydrazone moiety in the bicyclic derivative $\mathbf{6 k}$ was performed using concentrated hydrochloric acid and $38 \%$ solution of formaldehyde in THF affording aldehyde 8 in $95 \%$ yield. Subsequently, the incorporation of phenyl ring via Grignard reaction with phenylmagnesium bromide was performed providing 9 as a mixture of two diastereomers. The oxidation of the secondary alcohol $\mathbf{9}$ to the ketone $\mathbf{1 0}$ was accomplished in the next step by the treatment of 9 with 2,3-dichloro-5,6dicyano-1,4-benzoquinone (DDQ) in the presence of TEMPO. The deprotection of hydroxyl group in ketone $\mathbf{1 0}$ conducted under basic conditions and the oxidation of primary alcohol to carboxylic acid carried out with (diacetoxyiodo)benzene in the presence of TEMPO afforded enantiomerically pure ketorolac in a good yield. . Importantly, according to our knowledge, the developed protocol is the most enantioselective method described so far in the literature and constitutes a metal-free route to enantiomerically pure ketorolac consisting of 6 steps and was realized in good $22 \%$ overall yield.

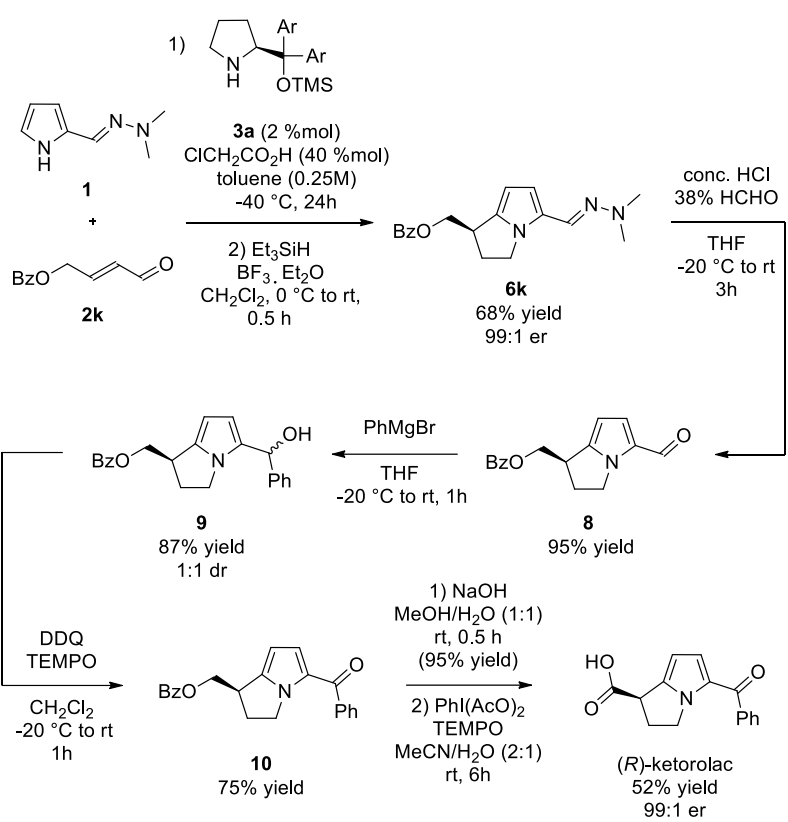

Scheme 4. Asymmetric organocatalytic synthesis of ketorolac.

In conclusion, an organocatalytic method for the construction of 2,3-dihydro- $1 \mathrm{H}$-pyrrolizine scaffold is described. It utilizes vinylogous reactivity of hydrazone derived from $1 \mathrm{H}$-pyrrole-2carbaldehyde that participates in a $(3+2)$-cycloaddition with $\alpha, \beta$ unsaturated aldehydes realized under aminocatalytic iminium ion activation. Target 2,3-dihydro-1 $H$-pyrrolizine were obtained with excellent chemical and stereochemical efficiency. The developed method was successfully employed in the first organocatalytic synthesis of ketorolac, a potent non-steroidal anti-inflammatory drug.

\section{Acknowledgements}

This project was realized within the Sheng programme (grant number: UMO-2018/30/Q/ST5/00466) from the National Science Centre, Poland. This contribution has been completed while the first author (JK) was the Doctoral Candidate in the Interdisciplinary Doctoral School at the Lodz University of Technology, Poland.

Keywords: organocatalysis • aminocatalysis • umpolung • ketorolac $\cdot$ total synthesis

[1] a) J. Hartwig, Nature 2005, 437, 487-488; b) Jacobsen, E.N.; Pfaltz, A.; Yamamoto, H. Comprehensive Asymmetric Catalysis: Supplement 1; Springer Science \& Business Media: Berlin/Heidelberg, Germany, 2003; Volume 1; c) M. Christmann, S. Bräse, Eds., Asymmetric Synthesis: The Essentials, Wiley-VCH: Weinheim, 2007.

[2] For selected reviews on organocatalysis, see: a) A. Erkkilä, I. Majander, P. M. Pihko, Chem. Rev. 2007, 107, 5416 - 5470; b) S. Mukherjee, J. W. Yang, S. Hoffmann, B. List, Chem. Rev. 2007, 107, 5471- 5569; c) P. Melchiorre, M. Marigo, A. Carlone, G. Bartoli, Angew. Chem. Int. Ed. 2008, 47, 6138 - 6138; d) M. Bella, T. Gasperi, Synthesis 2009, 1583 1614 ; e) D. Kampen, C. M. Reisinger, B. List, Top. Curr. Chem. 2010 291, 395 - 456; f) C. Palomo, M. Oiarbide, R. López, Chem. Soc. Rev. 2009, 38, 632 -653; g) L. Jiang, Y.-C. Chen, Catal. Sci. Technol. 2011, 
1, 354 - 365; h) J. Alemán, A. Parra, H. Jiang, K. A. Jørgensen, Chem. Eur. J. 2011, 17, 6890 - 6899; i) P. Melchiorre, Angew. Chem. Int. Ed 2012, 51, 9748 - 9770; j) C. Quigley, Z. Rodríguez-Docampo, S. J. Connon, Chem. Commun. 2012, 48, 1443 - 1445; k) M. Tsakos, C. G. Kokotos, Tetrahedron, 2013, 69, 10199 - 10222; I) M. Dzięgielewski, J. Pięta, E. Kamińska, Ł. Albrecht, Eur. J. Org. Chem. 2015, 677 - 702; m) H. Krawczyk, M. Dzięgielewski, D. Deredas, A. Albrecht, Ł. Albrecht, Chem. Eur. J. 2015, 21, 10268 - 10277; n) D. M. Flanigan, F. Romanov-Michailidis, N. A. White, T. Rovis, Chem. Rev. 2015, 115 9307 - 9387; o) G. Koutoulogenis, N. Kaplaneris, C. G. Kokotos Beilstein J. Org. Chem. 2016, 12, 462 - 495; p) J. Merad, J.-M. Pons, O Chuzel, C. Bressy, Eur. J. Org. Chem. 2016, 5589 - 5610; q) B. Teng, W. C. Lim, C.-H. Tan, Synlett 2017, 28, 1272-1277; r) H. Guo, Y. C Fan, Z. Sun, Y. Wu, O. Kwon, Chem. Rev. 2018, 118, 10049-10293.

[3] a) Carreira, L. Kvaerno,. Classics in Stereoselective Synthesis, Wiley$\mathrm{VCH}$, Weinheim, 2008

[4] a) D. Yi, T. Bayer, C. P. S. Badenhorst, S. Wu, M. Doerr, M. Höhne, U. T. Bornscheuer, Chem. Soc. Rev. 2021, 50, 8003-8049; b) T. Laird, Org. Process Res. Dev. 2009, 13, 656-656; c) J. Albarrán-Velo, D. González-Martínez, V. Gotor-Fernández, Biocatal. Biotransformation 2018, 36, 102-130.

[5] For selected reviews on asymmetric aminocatalysis, see: a) B. List, Chem. Commun. 2006, 819-824; b) A. Erkkilä, I. Majander, P. M. Pihko Chem. Rev. 2007, 107, 5416-5470; c) S. Mukherjee, J.-W. Yang, S. Hoffmann, B. List, Chem. Rev. 2007, 107, 5471-5569; d) P. Melchiorre, M. Marigo, A. Carlone, G. Bartoli, Angew. Chem., Int. Ed. 2008, 47 6138-6171; e) L. Jiang, Y.-C. Chen, Catal. Sci. Technol. 2011, 1, 354 365; f) P. Melchiorre, Angew. Chem., Int. Ed. 2012, 51, 9748-9770.

[6] a) T. Mukaiyama, H. Ishikawa, H. Koshino, Y. Hayashi, Chem. - Eur. J. 2013, 19, 17789-1780; b) Y. Hayashi, S. Ogasawara, Org. Lett. 2016 18, 3426-3429; c) F. Xu, M. Zacuto, N. Yoshikawa, R. Desmond, S Hoerrner, T. Itoh, M. Journet, G. R. Humphrey, C. Cowden, N. Strotman, P. Devine, J. Org. Chem. 2010, 75, 7829-7841; d) P. Szcześniak, S. Buda, L. Lefevre, O. Staszewska-Krajewska, J. Młynarski, Eur. J. Org. Chem. 2019, 6973-6982; e) N.A. Paras, B. Simmons, D.W.C. MacMillan, Tetrahedron 2009, 65, 3232-3238; f) A. Pou, A. Moyano, Eur. J. Org. Chem. 2013, 2013, 3103-3111.

[7] https://clincalc.com/DrugStats/Top300Drugs.aspx

[8] a) W. H. Rooks WH, A. J. Tomolonis, P. J. Maloney, M. B. Wallach, M. E. Schuler, Clin. Pharmacol. Ther. 1982, 2, 684-690 ; b) A. Guzmán, F. Yuste, R. A. Toscano, J. M. Young, A. R. Van Horn, J. M. Muchowski, J Med. Chem. 1986, 29, 589-591; c) H. J. McQuay, P. Poppleton, D. Carroll, R. J. Summerfield, R. E. Bullingham, R. A. Moore, Clin. Pharmacol. Ther. 1986, 39, 89-93.

[9] a) J. M. Muchowski, S. H. Unger, J. Ackrell, P. Cheung, G. F. Cooper, J. Cook, P. Gallegra, O. Halpern, R. Koehler, A. F. Kluge, A. R. Van Horn, Y. Antonio, H. Carpió, F. Franco, E. Galeazzi, I. Garcia, R Greenhouse, A. Guzman, J. Iriarte, A. Leon, A. Peña, V. Perez, D. Valdez, N. Ackerman, S. A. Bailaron, D. V. Krishna Murthy, J. R. Rovito A. J. Tomolonis, J. M. Young, W. H. Rooks, J. Med. Chem. 1985, 28, 1037-1049; b) D. R. Artis, I.-S. Cho, J. M. Muchowski, Can. J. Chem 1992, 70, 1838-842; c) E. Flórez-López, L. B. Gomez-Pérez, L. D. Miranda, Tetrahedron Lett. 2010, 51, 6000-6002; d) P. Malik, R. Bhushan, New J. Chem. 2017, 41, 13681-13691; e) Y. H. Kim, C. S Cheong, S. H. Lee, K. S. Kim, Tetrahedron: Asymmetry, 2001, 12, 1865-1869; f) M. Perez-Venegas, A. M. Rodriguez-Trevino, E. Juaristi, ChemCatChem 2020, 12, 1782-1788; g) P. S. Baran, J. M. Richter, D. W. Lin, Angew. Chem. Int. Ed. 2005, 44, 609-612; h) J. M. Richter, B. W. Whitefield, T. J. Maimone, D. W. Lin, M. P. Castroviejo, P. S. Baran, J. Am. Chem. Soc. 2007, 129, 12857-12869; i) I. Sasaki, N. Yamasaki, Y. Kasai, H. Imagawa, H. Yamamoto, Tetrahedron Lett. 2020, 61, 151564

[10] a) X. Bugaut, F. Glorius, Chem. Soc. Rev. 2012, 41, 3511-3522; b) D. M. Flanigan, F. Romanov-Michailidis, N. A. White, T. Rovis, Chem. Rev. 2015, 115, 9307-9387; c) R. S. Menon, A. T. Biju, V. Nair, Chem. Soc. Rev. 2015, 44, 5040-5052; d) R. S. Menon, A. T. Biju, V. Nair, Beilstein J. Org. Chem. 2016, 12, 444-461.

[11] For reviews on the hydrazone umpolung reactivity, see: a) R. Brehme, D. Enders, R. Fernandez, J. M. Lassaletta, Eur. J. Org. Chem. 2007,
5629-5660; b) M. de Gracia Retamosa, E. Matador, D. Monge, J. M. Lassaletta, R. Fernández, Chem. - Eur. J. 2016, 22, 13430-13445; For the recent utilization of hydrazone umpolung in organocatalytic approaches: c) K. Nagaraju, R. Gurubrahamam, K. Chen, J. Org. Chem. 2020, 85, 7060-7067; d) E. Matador, M. de Gracia Retamosa, D. Monge, R. Fernández, J. M. Lassaletta, Chem. Commun. 2020, 56, 9256-9267; e) M. Gómez-Martínez, M. del Carmen Pérez-Aguilar, D. G. Piekarski, C. G. Daniliuc, O. García Mancheño, Angew. Chem., Int. Ed. 2021, 60, 5096-5107; f) B. Łukasik, J. Kowalska, S. Frankowski, Ł. Albrecht, Chem. Commun. 2021, 57, 6312-6315.

[12] a) M. F. Jett, C. S. Ramesha, C. D. Brown, S. Chiu, C. Emmett, T. Voronin, T. Sun, C. O'Yang, J. C. Hunter, R. M. Eglen, R. M. Johnson, J. Pharmacol. Exp. Ther. 1999, 288, 1288-1297; b) I. C. Hsu, J. R. Allen, C. F. Chesney, Proc. Soc. Exp. Biol. Med. 1973, 144, 834-838; C) S. K. Kulkarni, V. P. Singh, 2007, 7, 251-263.

[13] CCDC 2136507 (6b) contains the supplementary crystallographic data for this paper. These data can be obtained free of charge from The Cambridge Crystallographic Data Centre via www.ccdc.cam.ac.uk/structures.

[14] (a) G. Lelais, D. W. C. MacMillan, Aldrichimica Acta, 2006, 39, 79-87; (b) R. Mahrwald, In Comprehensive Enantioselective Organocatalysis, ed. P. I. Dalko, 2013; (c) D. Seebach, U. Grošelj, D. M. Badine, W. B. Schweizer, A. K. Beck, Helv. Chim. Acta, 2008, 91, 1999-2034. 


\section{Entry for the Table of Contents}

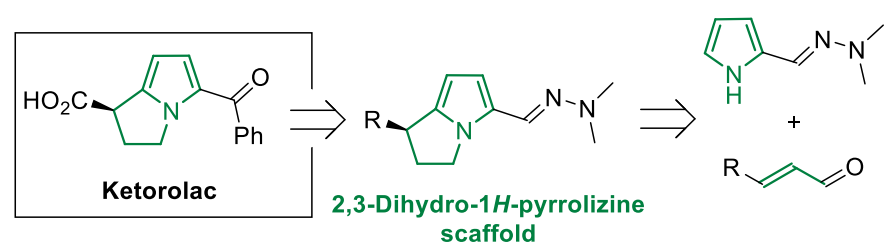

The manuscript describes the first organocatalytic synthesis of ketorolac, a potent non-steroidal anti-inflammatory drug. For this purpose, the organocatalytic (3+2)-cycloaddition between hydrazone derived from $1 \mathrm{H}$-pyrrole-2-carbaldehyde and $\alpha, \beta$-unsaturated aldehydes realized under aminocatalytic iminium ion activation was developed. It constitutes the key step of the method allowing for the construction of 2,3-dihydro-1 $\mathrm{H}$-pyrrolizine scaffold.

Institute and/or researcher Twitter usernames: @a_teamlab 